\title{
Courbes et efficacité biologique de croissance d'agneaux de différents types génétiques abattus à l'âge de 100 et $180 \mathrm{j}$
}

\author{
B Portolano *, M Todaro \\ Institut de zootechnie générale, faculté d'agronomie, université de Palerme, Viale delle Scienze, \\ Parco d'Orleans, 90128 Palerme, Italie
}

(Reçu le 28 novembre 1995 ; accepté le 9 décembre 1996)

\begin{abstract}
Summary - Curves and biological efficiency of growth for 100- and 180-day-old lambs of different genetic types. Within a MIP (Mediterranean integrated programs) research project, the effect of a crossbreed between Suffolk breed rams, and Comisana and Pinzirata breed ewes was evaluated. This effect was evaluated with the lambs' growth curves estimated with the semilogarithmic function proposed by Bettini (1987): $\mathrm{Y}(\mathrm{t})=a+b \mathrm{t}+c \ln (\mathrm{t})$. The experiment concerned 122 lambs of four genetic types: 44 Pinzirita $\times$ Pinzirita lambs, 42 Suffolk $\times$ Pinzirita lambs, 15 Comisana $\times$ Comisana lambs and 20 Suffolk $\times$ Comisana lambs. After the weaning (60 days), the lambs were fed with vetch hay and concentrate until days 100 and 180 of life. Besides, for the four genetic types $(\mathrm{S} \times \mathrm{C}$, $\mathrm{S} \times \mathrm{P}, \mathrm{C} \times \mathrm{C}, \mathrm{P} \times \mathrm{P}$ ), the biologic efficiency of growth $(\mathrm{BEG})$ was calculated. The $a, b$ and $c$ growth function parameters were higher for crossbreed lambs than for pure lambs and were higher for males than for females. These differences were greater for 180-day-old lambs than for 100-day-old lambs. The biologic efficiency of growth was higher for 100-day-old lambs than for 180-day-old lambs and it was greater for males than for females, independently from the genetic type. The BEG curves, distinguished by genetic type, showed that, after 32 days of life for the Comisana breed and after 65 days of life for the Pinzirita breed, it is more convenient to raise crossbreed lambs than pure lambs. The Suffolk breed ram, used for crossbreed with the Comisana and the Pinzirita breed ewes, improves the sheep meat production. The best results were obtained with 180-day-old lambs.
\end{abstract}

biologic efficiency of growth / growth curves / lambs / Comisana breed ewes / Pinzirita breed ewes / Suffolk breed ram

Résumé - Dans le cadre d'un projet conduit par les Pim (Programmes intégrés méditerranéens), nous avons mené une étude afin d'évaluer l'effet d'un croisement entre béliers Suffolk et brebis Comisana et Pinzirita dans les conditions d'élevage difficile des zones montagneuses typiques de la Sicile. Cette étude a été réalisée à l'aide des courbes de croissance des agneaux, estimées avec la fonction

* Correspondance et tirés à part 
semi-logarithmique de Bettini: $\mathrm{Y}(t)=a+b t+c \ln (t)$. En outre, l'efficacité biologique de croissance a été calculée pour les quatre types génétiques $(\mathrm{S} \times \mathrm{C}, \mathrm{S} \times \mathrm{P}, \mathrm{C} \times \mathrm{C}, \mathrm{P} \times \mathbf{P})$. Les paramètres $a, b$ et $c$ de la fonction de croissance ont été, en moyenne, plus élevés chez les métis que chez les sujets purs et chez les mâles que chez les femelles. Ces différences ont été plus marquées chez les agneaux abattus à $180 \mathrm{j}$ qu'à $100 \mathrm{j}$. L'efficacité biologique de croissance a été supérieure chez les agneaux de $100 \mathrm{j}$ et, indépendamment du type génétique, elle a été supérieure chez les mâles. Les indices de consommation, exprimés en kilo de matière sèche par kilo de gain de poids, ont été de 5,55 et 5,44 respectivement pour les types génétiques Pinzirita $\times$ Pinzirita et Suffolk $\times$ Pinzirita abattus à l'âge de $180 \mathrm{j}$. Pour les agneaux abattus à l'âge de $100 \mathrm{j}$, les indices de consommation ont été de 5,18 pour le type génétique Pinzirita $\times$ Pinzirita et de 5,09 pour le type génétique Suffolk $\times$ Pinzirita. Les courbes de l'efficacité biologique de croissance, avec les indices de consommation, réparties par type génétique, ont montré qu'après $32 \mathrm{j}$ pour la race Comisana et $65 \mathrm{j}$ pour la race Pinzirita il convient d'élever des sujets de croisement plutôt que des sujets purs.

efficacité biologique de croissance / courbe de croissance / agneau / brebis Comisana / Pinzirita / bélier Suffolk

\section{INTRODUCTION}

Les possibilités offertes par le croisement de première génération pour l'amélioration quantitative et qualitative de la production de viande des races ovines laitières méritent une analyse plus approndie dans les conditions difficiles des zones montagneuses de la Sicile. La plupart des travaux entrepris sur les races ovines laitières de la Sicile ont fourni, en effet, beaucoup d'informations sur les performances de production des animaux obtenus par le croisement de première génération avec les béliers des principales races à viande étrangères (Galvano et Lanza, 1976; Lanza et al, 1982a, b) et italiennes (Chiofalo, 1982 ; Chiofalo, 1983 ; Lanza et al, 1983), en conditions d'élevage optimal. En revanche, il existe peu d'informations sur les résultats qui pourraient être obtenus dans les systèmes laitiers traditionnels, pour lesquels la production de viande, à partir des brebis reformées et des agneaux excédentaires constitue un revenu supplémentaire.

Dans le cadre d'un vaste projet de recherche conduit par les PIM (programmes intégrés méditerranéens), sensibles aux problématiques inhérentes à l'augmentation de la production de viande ovine dans le centre de la Sicile, l'Institut de zootechnie générale de la faculté d'agronomie de l'université de Palerme a mené une étude pour évaluer l'effet des croisements Suffolk $\times$ Comisana et Suffolk $\times$ Pinzirita sur la production d'agneaux à abattre à l'âge de 100 et $180 \mathbf{j}$.

L'objectif de ce travail a été d'évaluer les résultats en fonction des informations obtenues à partir des courbes de croissance des agneaux de quatre types génétiques, courbes définies par le modèle suivant: $\mathrm{Y}_{(t)}$ $=a+b t+c \ln (t)$ (Bettini, 1987), et d'étudier l'effet du type génétique sur l'efficacité biologique de croissance.

\section{MATÉRIEL ET MÉTHODES}

L'étude a été réalisée dans deux exploitations possédant des brebis de race Comisana et Pinzirita, sur 121 agneaux de quatre types génétiques : 44 de race Pinzirita, 42 Suffolk $\times$ Pinzirita, 15 de race Comisana et 20 Suffolk $\times$ Comisana des deux sexes, nés en août-septembre 1994. En ce qui concerne les types génétiques de la race Comisana, la catégorie des agneaux de $180 \mathrm{j}$ a été éliminée car leur nombre n'était pas suffisant pour l'analyse statistique.

Après la naissance des agneaux, deux lots correspondant aux catégories de 100 et $180 \mathrm{j}$ ont été formés. Ils ont été allaités jusqu'au sevrage à $60 \mathrm{j}$. Après le sevrage, tous les agneaux ont reçu 
à volonté une ration composée de foin de vesce (MAT 179 et CB $227 \mathrm{~g} / \mathrm{kg}$ de matière sèche) et d'un aliment concentré équilibré (MAT $17 \mathrm{l}$ et CB $91 \mathrm{~g} / \mathrm{kg}$ de matière sèche). Une fois par semaine, jusqu'à l'abattage, le poids vif a été mesuré.

\section{Analyse statistique}

Les effets de croisement entre bélier de race Suffolk et brebis de races Comisana et Pinzirita ont été étudiés en comparant les courbes de croissance des agneaux, obtenues en utilisant la fonction suivante : $\mathrm{Y}_{(t)}=a+b t+c \ln (t)$ [1] (Bettini, 1987) où $Y_{(r)}=$ poids vif des agneaux en $\mathrm{kg}$; $t=$ âge des agneaux en jours ; $a, b$, et $c=$ paramètres à estimer de la courbe de croissance.

Cette fonction a été choisie car, contrairement à d'autres modèles (Brody, 1921 ; Richards, 1959 ; Koops, 1986 ; Koops et Grossman, 1991), elle ne nécessite pas de connaître la valeur asymptotique du poids vif, c'est-à-dire le poids vif à l'âge adulte. Dans l'étude présente, cette valeur n'était pas disponible, les agneaux ayant été abattus à l'âge maximum de 6 mois.

Les paramètres $a, b$ et $c$ de la courbe de croissance ont été estimés par régression multiple, pour ajuster cette courbe aux données hebdomadaires du poids de chaque agneau. $L$ 'analyse de variance sur les coefficients estimés a été de la forme suivante : $\mathrm{Y}_{i j l k}=m+\mathrm{TG}_{i}+\mathrm{S}_{j}+\mathrm{A}_{l}$ $+(\mathrm{TG} * \mathrm{~S} * \mathrm{~A})_{i j l}+\mathrm{e}_{i j / k}[2]$ où $\mathrm{Y}_{i j / k}=$ paramètres de la courbe ; $\mathrm{TG}_{\mathrm{i}}=$ type génétique, $i=1,2$; $\mathrm{S}_{j}=$ sexe des agneaux, $j=1,2 ; \mathrm{A}_{l}=$ âge à l'abattage, $l=1,2 ; \mathrm{TG}^{*} \mathrm{~S}^{*} \mathrm{~A}=$ interaction entre les trois facteurs $; \mathrm{e}_{i j l k}=$ résidu.

Pour chaque combinaison type génétique $x$ sexe $\times$ âge, les accroissements de poids à intervalles de $10 \mathrm{j}$ ont été déterminés en résolvant la dérivée première de la fonction $\mathrm{Y}_{(t)}=a+b t+c$ $\ln (t)$. La moyenne de ces valeurs a fourni le gain moyen quotidien du poids vif à partir du dixième jour jusqu'à l'âge d'abbattage.

L'efficacité biologique de croissance a été exprimée par le rapport entre le gain de poids (en g) et le poids vif en $\mathrm{kg}$ de poids métabolique $\left(\mathrm{PV}^{\mathbf{0}, 75}\right)$ dans un laps de temps défini. Elle mesure le rendement dans des conditions de milieu et de conduite d'élevage (Pilla et al, 1987) determinées. Les valeurs ainsi obtenues ont été soumises à l'analyse de variance avec le modèle [2]. En outre, l'évolution de l'efficacité biologique au cours de l'expérience a également été estimée à l'aide d'une fonction exponentielle décroissante du type : $\mathrm{Y}=a e^{-b t}[3]$ où $\mathrm{Y}_{(t)}=$ efficacité biologique de croissance à l'âge $t ; t=$ âge des agneaux en jours ; $a$ et $b=$ paramètres à estimer de la fonction [3]. À partir des données réelles de chaque agneau, les paramètres $a$ et $b$ de la fonction [3] ont été estimés avec un algorithme de régression non linéaire (Marquardt, 1963). Le critère de convergence utilisé a été : $\left(\mathrm{SSE}_{i-1}-\mathrm{SSE}_{i}\right) /\left(\mathrm{SSE}_{i}\right.$ $\left.+10^{-6}\right)<10^{-8}$ où $\mathrm{SSE}_{i}$ est la déviance de l'erreur de l'ième itération. À partir de ces paramètres le point de rencontre des courbes des différents types génétiques a été calculé avec le rapport suivant : $x=\frac{\ln \left(a_{i}\right)-\ln \left(a_{i}\right)}{\left(b_{i}-b_{i}\right)}[5]$ où $i$ et $i^{\prime}$ indiquent les niveaux des facteurs ou des combinaisons (Pilla et al 1987).

Toutes les confrontations entre les moyennes ont été effectuées avec le test $t$ de Student. L'analyse statistique a été effectuée avec les procédures GLM, REG et NLIN du software statistique Sas 6.09.

\section{RÉSULTATS ET DISCUSSION}

Le tableau I donne les valeurs moyennes estimées des coefficients de la courbe de croissance pour les agneaux de $100 \mathrm{j}$. Les valeurs de $R^{2}$ sont élevées, comprises entre 0,76 et 0,91 et montrent l'excellente adaptation de la courbes aux données expérimentales. Entre les types génétiques, des différences statistiquement significatives sont obtenues chez les mâles seulement, en particulier, entre Pinzirita purs et Suffolk $\times$ Pinzirita pour les paramètres $a(4,99$ vs $6,20 ; p \leq 0,05) ; b(0,16$ vs 0,$21 ; p \leq 0,01)$ et entre Comisana purs et Suffolk $\times$ Comisana pour le paramètre $c(1,05$ vs 0,17 ; $p \leq 0,01)$. En ce qui concerne le facteur « sexe », des différences significatives sont mises en évidence pour le paramètre $c$ chez le type génétique Suffolk $\times$ Comisana $(0,17$ vs 0,$69 ; p \leq 0,01)$ et pour les paramètres $a$, $b$ et $c$ chez le type génétique Suffolk $\times$ Pinzirita $(6,20$ vs 4,$43 ; p \leq 0,01 ; 0,21$ vs 0,16 ; $p \leq 0,01$ et 0,39 vs 0,$74 ; p \leq 0,05$ respectivement pour $a, b$ et $c$ ). 
Le tableau II donne les valeurs moyennes estimées des coefficients $a, b$ et $c$ des agneaux de 180 jours pour les types génétiques Pinzirita $\times$ Pinzirita et Suffolk $\times$ Pinzirita. Pour ces agneaux, les valeurs du coefficient de détermination $R^{2}$ sont aussi élevées et comprises entre 0,909 et 0,888 . Comme pour la catégorie précédente, des différences significatives entre types génétiques sont mises en évidence chez les mâles pour le paramètre $b(0,185$ vs 0,228 ; $p \leq 0,01)$. Les différences entre sexes, au sein d'un même type génétique, sont observées respectivement pour les paramètres $b$ et $c: 0,185$ vs 0,$145 ; p \leq 0,05$ et 0,422 vs 0,$525 ; p \leq 0,01$ pour le type génétique $\mathrm{Pin}$ zirita $\times$ Pinzirita et 0,228 vs 0,$169 ; p \leq 0,01$ et 0,778 vs 0,$152 ; p \leq 0,01$ pour le type génétique Suffolk $\times$ Pinzirita). Les résultats obtenus mettent en évidence que l'évolution différente de la croissance entre les types génétiques et l'âge d'abattage n'est notable que chez les mâles. L'effet du dimorphisme sexuel se manifeste plus chez les types génétiques à base maternelle Pinzirita que Comisana.

Les gains moyens de poids vif en $\mathrm{g} / \mathrm{j}$ (GMQ) par type génétique, sexe et âge d'abattage ont été calculés à partir de la fonction semi-logarithmique de Bettini (tableau III).

Les femelles de $100 \mathrm{j}$, du type génétique Comisana $\times$ Comisana, ont eu des GMQ significativement inférieurs à ceux des agnelles Suffolk $\times$ Comisana (168 vs $202 \mathrm{~g} / \mathrm{j} ; p \leq 0,01$ ), mais il n'y a pas eu de différence significative chez les mâles (221 vs $214 \mathrm{~g} / \mathrm{j}$ ). En revanche entre les sujets purs Pinzirita $\times$ Pinzirita et les croisés Suffolk $\times$ Pinzirita, les mâles croisés ont eu des GMQ supérieurs aux purs (216 vs $185 \mathrm{~g} / \mathrm{j}$; $p \leq 0,01)$ alors qu'aucune différence n'a été notée chez les femelles. Le facteur « sexe », au sein d'un type génétique, est toujours évident sauf chez les sujets Pinzirita $\times$ Pinzirita où les mâles ont eu des GMQ comparables à ceux des femelles. Chez les sujets abattus à $180 \mathrm{j}$, les effets des facteurs «sexe » et «type génétique » sont beaucoup plus nets. En effet, ce sont toujours les agneaux croisés qui ont eu les GMQ les plus élevés, que ce soit chez les mâles (213 vs $177 \mathrm{~g} / \mathrm{j} ; p \leq 0,01)$ ou chez les femelles ( 172 vs $155 \mathrm{~g} / \mathrm{j} ; p \leq 0,01)$.

Les consommations d'aliment et les indices de consommation, du sevrage au jour 100 ou 180 (kg de MS consommée par $\mathrm{kg}$ de gain de poids vif) sont données dans le tableau IV. En ce qui concerne les types génétiques à base maternelle Comisana, les consommations d'aliment sont plus importantes chez les sujets purs, alors que l'indice de consommation est plus faible chez les sujets croisés. Chez les types génétiques à base maternelle Pinzirita, les consommations d'aliments et l'indice de consommation sont peu différents. De l'ensemble de ces données, on peut conclure qu'à $100 \mathrm{j}$ d'âge les résultats ne sont pas uniformes pour les différents types génétiques. La race de la mère (Pinzirita et Comisana) et le mode de gestion du troupeau jouent probablement un rôle de premier plan dans la croissance des jeunes agneaux. Au contraire, chez les sujets âgés de $180 \mathrm{j}$, les résultats sont plus uniformes. Il est probable qu'au fur et à mesure que les agneaux vieillissent les caractéristiques typiques de la race à viande croisée Suffolk réussissent à mieux s'exprimer.

L'analyse de l'efficacité biologique de croissance du poids vif met en évidence, indépendamment du type génétique, une supériorité des mâles sur les femelles. En particulier, pour les agneaux de $100 \mathrm{j}$ (tableau V), il y a des différences très significatives entre les sexes. Dans la même catégorie d'agneaux, il n'y a des différences entre types génétiques que pour ceux de base maternelle Comisana en faveur des croisés tant pour les mâles et les femelles.

De même pour les agneaux âgés de $180 \mathrm{j}$ (tableau VI), l'efficacité des mâles est supérieure à celle des femelles. En ce qui 

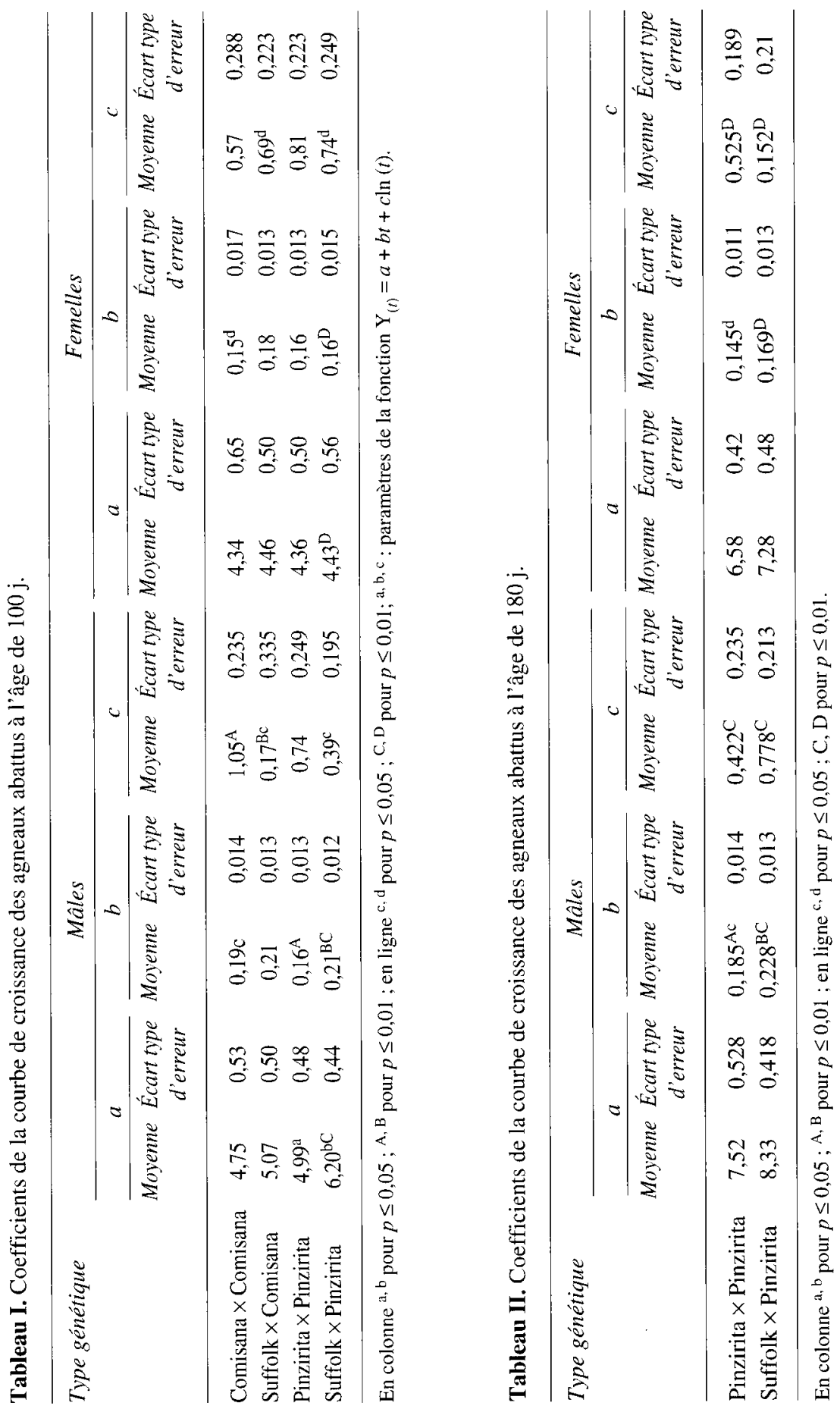


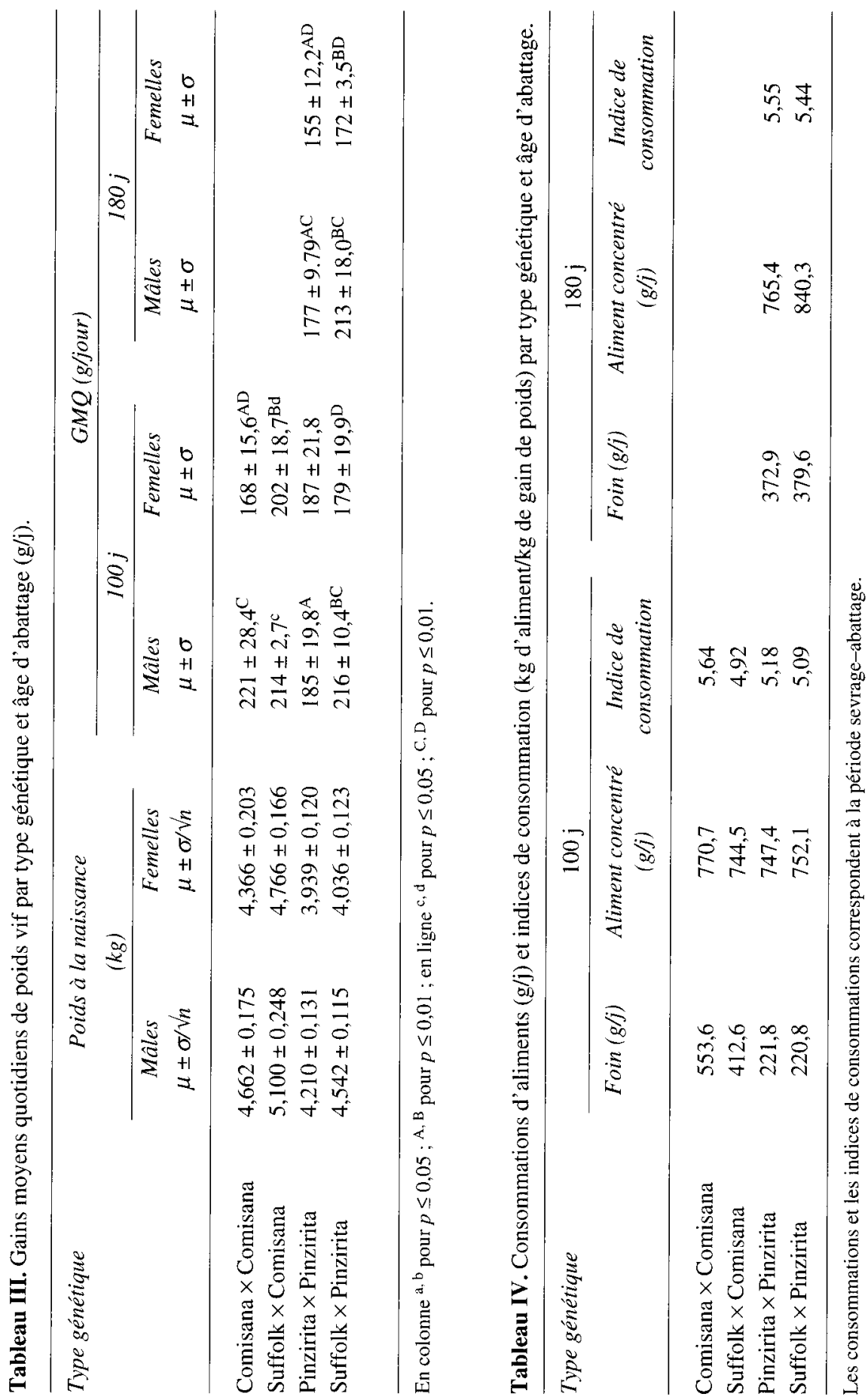


Tableau V. Efficacité biologique du gain de poids vif par type génétique et sexe chez les agneaux de $100 \mathrm{j}$ (g de gain de poids vif/kg de $\mathrm{P}^{0,75}$ ).

\begin{tabular}{|c|c|c|c|c|}
\hline \multirow[t]{3}{*}{ Type génétique } & \multicolumn{4}{|c|}{$100 j$} \\
\hline & \multicolumn{2}{|c|}{ Mâles } & \multicolumn{2}{|c|}{ Femelles } \\
\hline & Moyenne & Ecart type d'erreur & Moyenne & $\bar{E}$ cart type d'erreur \\
\hline Comisana $\times$ Comisana & $18,09^{\mathrm{aC}}$ & 0,93 & $16,73^{\mathrm{AD}}$ & 1,07 \\
\hline Suffolk $\times$ Comisana & $19,32^{\mathrm{bC}}$ & 1,31 & $17,73^{\mathrm{BD}}$ & 0,87 \\
\hline Pinzirita $\times$ Pinzirita & $17,96^{\mathrm{C}}$ & 0,57 & $16,96^{\mathrm{D}}$ & 0,53 \\
\hline Suffolk $\times$ Pinzirita & $18,71^{\mathrm{C}}$ & 0,56 & $16,68^{\mathrm{D}}$ & 0,60 \\
\hline
\end{tabular}

En colonne ${ }^{\mathrm{a} . \mathrm{b}}$ pour $p \leq 0,05 ;{ }^{\mathrm{A}, \mathrm{B}}$ pour $p \leq 0,01$; en ligne ${ }^{\mathrm{c} . \mathrm{d}}$ pour $p \leq 0,05 ;{ }^{\mathrm{C}, \mathrm{D}}$ pour $p \leq 0,01$.

Tableau VI. Efficacité biologique du gain de poids vif par type génétique et sexe chez les agneaux de $180 \mathrm{j}$ (g de gain de poids vif/kg de $\mathrm{P}^{0.75}$ ).

\begin{tabular}{lccccc}
\hline Type génétique & \multicolumn{3}{c}{$180 j$} & \\
\cline { 2 - 3 } \cline { 5 - 6 } & Moyenne & Écart type d'erreur & & \multicolumn{2}{c}{ Moyenenelles } \\
\hline Pinzirita $\times$ Pinzirita & $12,25^{\mathrm{c}}$ & 0,30 & & $11,51^{\text {ad }}$ & 0,26 \\
Suffolk $\times$ Pinzirita & $12,89^{\mathrm{c}}$ & 0,28 & & $12,06^{\mathrm{bd}}$ & 0,30 \\
\hline
\end{tabular}

En colonne ${ }^{\text {a.b }}$ pour $p \leq 0,05 ;{ }^{A, B}$ pour $p \leq 0,01$; en ligne ${ }^{c, d}$ pour $p \leq 0,05 ;{ }^{C}, \mathrm{D}$ pour $p \leq 0,01$.

concerne le type génétique, il y a des différences significatives entre femelles Pinzirita purs et les croisés Suffolk $\times$ Pinzirita.

En définitive, l'efficacité biologique a été plus élevée chez les mâles que chez les femelles, chez les sujets métis que chez les sujets purs, et, comme il était logique de le prévoir, chez les sujets âgés de $100 \mathrm{j}$ que chez ceux âgés de $180 \mathrm{j}$, même si parfois les différences n'ont pas été statistiquement significatives.

La figure 1 donne l'évolution de l'efficacité biologique de croissance en fonction de l'âge des agneaux. L'examen du graphique met en évidence, en accord avec ce qu'affirment Pilla et al (1987), que, plus la valeur initiale de l'efficacité est élevée, plus celle-ci décrô̂t rapidement avec l'âge. En outre, on peut dire qu'à des évolutions plus linéaires, c'est-à-dire avec des valeurs initiales plus basses et une baisse modérée, correspondent des efficacités biologiques de croissance globalement plus éle vées. La figure montre aussi à quel âge l'efficacité biologique de croissance d'un type génétique donné descend au dessous de celle d'un autre type génétique ; le point de rencontre entre les courbes des agneaux Comisana $\times$ Comisana et Suffolk $\times$ Comisana se situe à $32 \mathrm{j}$, alors que, pour les types génétiques Pinzirita $\times$ Pinzirita et Suffolk $\times$ Pinzirita, il se situe à $65 \mathrm{j}$. À partir de cet âge, l'efficacité des purs devient donc inférieure 


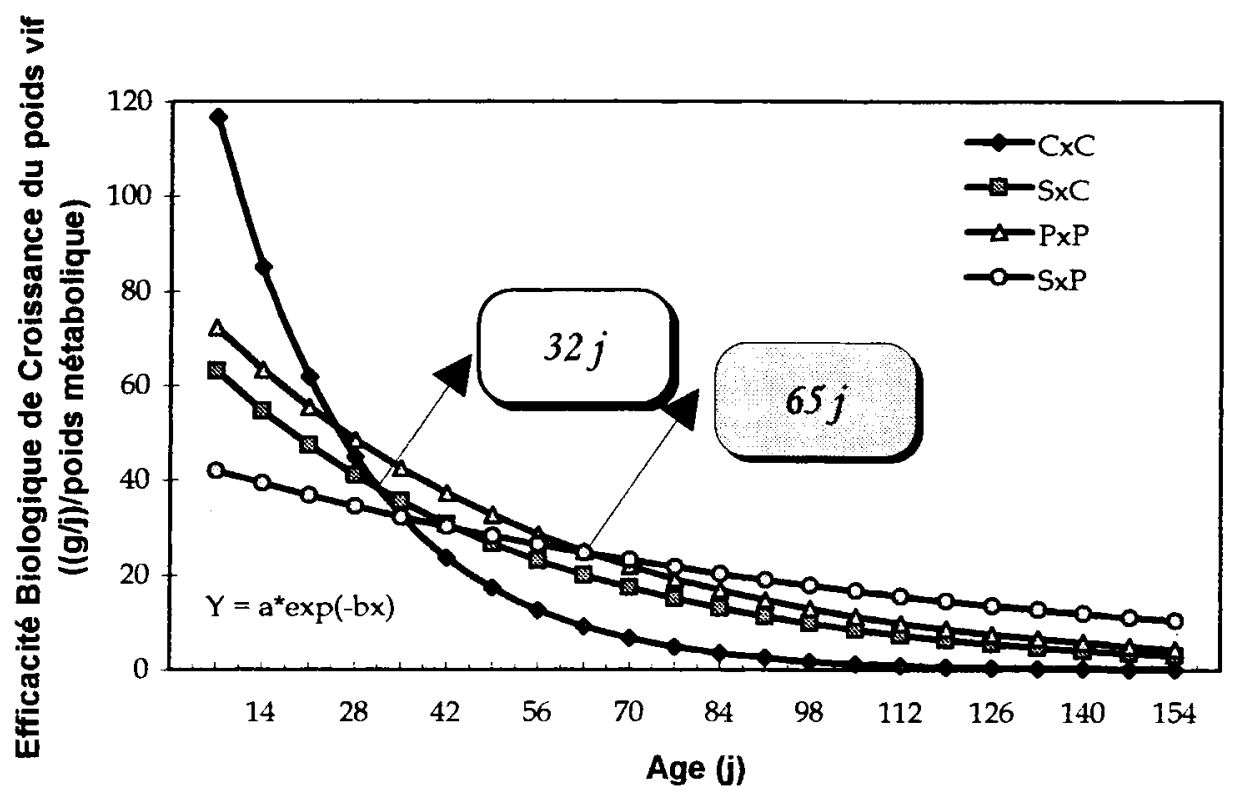

Fig 1. Évolution de l'efficacité biologique du gain de poids vif des agneaux appartenant aux différents types génétiques.

à celle des croisés. La meilleure efficacité biologique de croissance des croisés par rapport aux sujets purs est obtenue quand l'âge et donc le poids vif augmentent.

En définitive, le paramètre de l'efficacité biologique de croissance confirme qu'il est souhaitable d'abattre les croisés à des âges supérieurs à ceux pratiqués dans notre expérience. Cependant si on prend en compte les données d'abattage et de la dissection de la moitié de la carcasse de ces agneaux obtenus dans d'autres travaux (Giaccone et al, 1995 ; Portolano et al, 1995a,b), cela ne doit pas être étendu aux femelles pour éviter les carcasses trop grasses.

\section{CONCLUSION}

De cette étude, il peut être conclu que l'emploi des béliers de race Suffolk n'a pas toujours donné des résultats satisfaisants chez les sujets abattus à $100 \mathrm{j}$, car ces résultats ne sont pas toujours uniformes, en termes de gain de poids journalier, que ce soit entre types génétiques ou sexes. Au contraire, la situation est beaucoup plus nette pour les sujets abattus à l'âge de $180 \mathrm{j}$, pour lesquels la supériorité des croisés en termes d'accroissement journalier du poids est évidente.

Les animaux croisés ont un indice de consommation légèrement plus faible par rapport aux animaux de race pure pour le type génétique Suffolk $\times$ Comisana.

En termes d'efficacité biologique de croissance, la supériorité des croisés s'est manifestée chez les deux catégories d'agneaux ( 100 et $180 \mathrm{j}$ ). Leurs valeurs d'efficacité biologique de croissance sont toujours supérieures et le restent au cours de la période prise en considération. En 
conclusion, on peut affirmer, dans des conditions d'élevage extensif d'ovins à lait, que les béliers de race Suffolk peuvent améliorer la production de viande ovine, et que des résultats probablement meilleurs pourraient être obtenus en abattant les mâles croisés à un âge supérieur à $180 \mathrm{j}$.

\section{REMERCIEMENTS}

Nous remercions pour leur collaboration à la réalisation de ce travail, les techniciens S Spataro, A Gabriele et N Lo Bue de l'Institut de zootechnie générale de la faculté d'agronomie de l'université de Palerme.

Ce travail a été exécuté grâce au financement PIM de la Région sicilienne, sous-projet Agriculture.

\section{RÉFÉRENCES}

Bettini TM (1987) Elementi di scienze delle produzioni animali. Edagricole, Bologne, Italie, $1008 \mathrm{p}$

Brody $S$ (1921) The rate of growth of the dairy cow. $J$ Gentile Phyviol 3, 765-769

Chiofalo L (1982) Prove di incrocio tra aricti Barbareschi e Bergamaschi e pecore Siciliane per l'incremento della produzione di carne. Primi risultati agnelli da latte. Zoot Nutr Anim 8, 467-475

Chiofalo L (1983) Prove di incrocio fra ariete Bergamasco e pecora Siciliana. Produzione dell'agnellone leggero. In : Atti $5^{\circ}$ Congresso ASPA, Gargnano del Garda, Brescia, 4-9 juin 1983. Associazione Scientifica di Produzione Animale, Bologne, 357-364

Galvano G, Lanza A (1976) Effetti dell'incrocio lle de France $\times$ Siciliana e Texel $\times$ Siciliana sulla produzione dell'agnellone leggero. In : Atti $2^{\circ}$ Congresso ASPA Bari, 17-20 mai 1976. Associazione Scientifica di Produzione Animale, Bologne, 266-274
Giaccone P, Portolano B, Todaro M, Leto G, Bonanno A (1995) L'incrocio Suffolk $\times$ Pinzirita per la produzione di agnelli macellati a tre diverse età. Nota 2 : Rilievi "post mortem" In : Atti IXL Congresso SISVet Salsomaggiore Terme, Parma, 27-30 septembre 1995. Società Italiana delle Scienze Veterinarie, Messine

Koops WJ (1986) Multiphasic growth curve analysis. Growth 50, 160-165

Koops WJ, Grossman M (1991) Applications of a multiphasic growth function to body composition in pigs. J Anim Sci 69, 3265-3273

Lanza A, D'Urso G, Lanza E, Aleo C (1982a) Produzione dell agnellone Merinolandschaf $\times$ Siciliana di 130 giorni di età. Zoot Nutr Anim 8, 547-555

Lanza A, Mordechai M, Lanza E (1982b) Effetti dell'incrocio Berrichon du Cher x Comisana sulla produzione di carne ovina. I. Produzione dell'agnellone di 130 giorni di età. Zoot Nutr Anim 8, 565-574

Lanza A. Mordechai M. Lanza E (1983) Produzione dell agnellone Barbaresca $\times$ Comisana di 130 giomi di età. Zoot Nutr Anim 9, 49-59

Marquardt DW (1963). An algorithm for least-squares estimation of non linear parameters. $J$ Soc Indust Appl Math 11.431-441

Pilla AM, Catillo G, Gigli S, Romita A (1987) Confronto fra vitelloni meticci (Chianini, Charolaises, Limousines Marchigiano, Piemontesi e Romagnoli) e puri su Base materna Frisona. II Efficienza biologica dell accrescimento. Ann Ist Sper Zoot 20 (S.S.2), 27-44

Portolano B, Todaro M, Bonanno A, Leto G (1995a) Effetto dell' incrocio tra l'ariete Suffolk e la pecora Comisana per la produzione dell'agnello da latte e dell'agnello leggero. L'allevatore di ovini e caprini (sous presse)

Portolano B., Todaro M, Leto G, Alabiso M, Alicata ML ( 1995b) L'incrocio Suffolk $\times$ Pinzirita per la produzione di agnelli macellati a tre diverse età. Nota I. Rilievi in vita. In : Atti IXL Congresso SISVet. Salsomaggiore Terme, Parma, 27-30 septembre 1995. Società Italiana delle Scienze Veterinarie, Messine

Richards JF (1959) A flexible growth function for empirical use. $J$ Exp Bot 10, 290-300

Sas (1989) Sas/Stat User"s Guide, Volume 2. Sas Institute Inc, Cary, États-Unis, 846 p 